\title{
Cavitation flow simulation for a centrifugal pump at a low flow rate
}

\author{
TAN Lei ${ }^{1}$, ZHU BaoShan $^{2 *}$, CAO ShuLiang ${ }^{2} \&$ WANG YuMing ${ }^{1}$ \\ ${ }^{1}$ State Key Laboratory of Tribology, Tsinghua University, Beijing 100084, China; \\ ${ }^{2}$ State Key Laboratory of Hydroscience and Engineering, Tsinghua University, Beijing 100084, China
}

Received September 22, 2012; accepted October 25, 2012

\begin{abstract}
A computational model on the basis of the combination of the full cavitation model and the modified re-normalization group (RNG) $k-\varepsilon$ turbulence model are used to simulate the cavitating flow in a centrifugal pump at a low flow rate. A filter function is introduced for the RNG $k-\varepsilon$ turbulence model in order to consider the compressibility in the cavitating flow. The calculated values of the net positive suction head available $\left(H_{\mathrm{NPSH}}\right)$ agree well with the experimental measurements. The cavities in the impeller are obtained and their evolvement with $H_{\mathrm{NPSHa}}$ are revealed.
\end{abstract}

filter function, cavitation, computational model, centrifugal pump

Citation: Tan L, Zhu B S, Cao S L, et al. Cavitation flow simulation for a centrifugal pump at a low flow rate. Chin Sci Bull, 2013, 58: 949-952, doi: $10.1007 / \mathrm{s} 11434-013-5672-\mathrm{y}$

Cavitation is a very common physical phenomenon in pumps. It induces pressure fluctuation and uneven load distribution, and then seriously reduces pumps' efficiency and affects their stable operation ranges. In addition, the micro jets and impact forces due to collapse of the cavities can erode the surface of the flow passage components and damage the impeller blades. The service life of the pumps is then reduced greatly $[1,2]$. Due to the importance of the cavitation phenomenon and the great process in the cavitation and turbulence models, numerical simulations have been widely used to investigate the cavitation flow field in the pumps [3-6].

By using the cavitation model proposed by Kunz et al. [7], Medvitz et al. [3] calculated the cavitating flow in a centrifugal pump at small cavitation numbers. They analysed the reason why the pump head drops at small cavitation numbers for the off-designed condition, but the assumption of the potential flow in their calculations limits their methods used in more application. Coutier-Delgosha et al. [4] experimentally and numerically investigated the pressure distribution and hydraulic performance for a centrifugal pump with two-dimensional curved blade. Pouffary et al. [5]

*Corresponding author (email: bszhu@ mail.tsinghua.edu.cn) used the cavitation model based on the barotropic state law to accomplish the cavitation simulation for a centrifugal pump. Their results showed that when the sheet cavitation develops at the narrowest position between two blades, the pump's hydraulic characteristics decrease suddenly. Ding et al. [6] adopted the full cavitation model [8] to simulate the cavitating flow in a centrifugal pump. The critical values by their calculation matched well with experiments.

The mechanisms for cavitation have been extensively investigated $[9,10]$, and noticeable efforts have been made on the cavitaion and turbulence models for the simulation of cavitating flow [4]. However, it is difficult to predict the cavitating flow in pumps accurately, especially for off-designed conditions. In the present paper, we present a computational model for the cavitating flow in a centrifugal pump at a low off-design flow rate. The calculated results agree with the experimental data very well, which means that the computational model used is suitable for the cavitating flow simulation in the pumps.

\section{Mathematical model and numerical algorithm}

The fluid in the cavitating flow field for the pumps is con- 
sidered as a homogeneous and compressible mixed medium of vapor and liquid. The continuity and momentum equations in the Cartesian coordinates are as follows:

$$
\begin{gathered}
\frac{\partial \rho_{\mathrm{m}}}{\partial t}+\frac{\partial\left(\rho_{\mathrm{m}} u_{i}\right)}{\partial x_{i}}=0, \\
\frac{\partial\left(\rho_{\mathrm{m}} u_{i}\right)}{\partial t}+\frac{\partial\left(\rho_{\mathrm{m}} u_{i} u_{j}\right)}{\partial x_{j}}= \\
-\frac{\partial p}{\partial x_{i}}+\frac{\partial}{\partial x_{i}}\left[\left(\mu_{\mathrm{m}}+\mu_{t}\right)\left(\frac{\partial u_{i}}{\partial x_{j}}+\frac{\partial u_{j}}{\partial x_{i}}-\frac{2}{3} \frac{\partial u_{k}}{\partial x_{k}} \delta_{i j}\right)\right],
\end{gathered}
$$

where $\rho_{\mathrm{m}}$ and $\mu_{\mathrm{m}}$ are the mixture density and dynamic viscosity, calculated by weighted average of each phase volume fraction, $u$ is the velocity, $p$ is the pressure, and $\mu_{\mathrm{t}}$ is the turbulent viscosity, respectively. Subscripts $i, j, k$ denote the axes directions.

The phases change vigorously in the cavitating flow, leading to a great gradient of density in cavitation. Here, the full cavitation model proposed by Singhal et al. [8] is employed, and the mixture density is defined as

$$
\begin{gathered}
\frac{1}{\rho_{\mathrm{m}}}=\frac{f_{\mathrm{v}}}{\rho_{\mathrm{v}}}+\frac{f_{\mathrm{g}}}{\rho_{\mathrm{g}}}+\frac{f_{1}}{\rho_{\mathrm{l}}}, \\
f_{\mathrm{v}}=\frac{\alpha_{\mathrm{v}} \rho_{\mathrm{v}}}{\rho_{\mathrm{m}}}, f_{\mathrm{g}}=\frac{\alpha_{\mathrm{g}} \rho_{\mathrm{g}}}{\rho_{\mathrm{m}}}, f_{l}=\frac{\alpha_{1} \rho_{1}}{\rho_{\mathrm{m}}}=1-f_{\mathrm{v}}-f_{\mathrm{g}},
\end{gathered}
$$

where $f_{\mathrm{v}}, f_{\mathrm{g}}, f_{\mathrm{l}}$ are the component mass fractions of the vapor, gas, liquid, $\rho_{\mathrm{v}}, \rho_{\mathrm{g}}, \rho_{\mathrm{l}}$ are the component densities, and $\alpha_{\mathrm{v}}, \alpha_{\mathrm{g}}$, $\alpha_{1}$ are the component volume fractions. The vapor generation and condensation rates are modeled as follows:

$$
\begin{aligned}
& \frac{\partial}{\partial t}\left(\alpha_{\mathrm{v}} \rho_{\mathrm{v}}\right)+\nabla \cdot\left(\alpha_{\mathrm{v}} \rho_{\mathrm{v}} u\right)= \\
& C_{\mathrm{e}} \frac{\sqrt{k}}{\lambda} \rho_{1} \rho_{\mathrm{v}}\left[\frac{2}{3} \frac{p_{\mathrm{v}}-p}{\rho_{\mathrm{l}}}\right]^{1 / 2}\left(1-f_{\mathrm{v}}-f_{\mathrm{g}}\right) \\
& -C_{\mathrm{c}} \frac{\sqrt{k}}{\lambda} \rho_{1} \rho_{\mathrm{l}}\left[\frac{2}{3} \frac{p-p_{\mathrm{v}}}{\rho_{\mathrm{l}}}\right]^{1 / 2} f_{\mathrm{v}},
\end{aligned}
$$

where the empirical factors are $C_{\mathrm{e}}=0.02$ and $C_{\mathrm{c}}=0.01$, the gas mass fractions $f_{\mathrm{g}}=1.5 \times 10^{-5}$, the surface tension coefficient $\lambda=0.0717 \mathrm{~N} / \mathrm{m}, p_{\mathrm{v}}$ denotes vaporization pressure, and $k$ denotes turbulent kinetic energy.

The RNG $k-\varepsilon$ turbulence model, which makes some corrections on the model coefficients in the $\varepsilon$ equation of standard $k-\varepsilon$ turbulence model and can predict the rotating and curvature flow more accurately, is widely applied in fluid machinery. For RNG $k$ - $\varepsilon$ turbulence model, the $k$ and $\varepsilon$ equations can be written as:

$$
\begin{gathered}
\frac{\partial\left(\rho_{\mathrm{m}} u_{i} k\right)}{\partial x_{i}}=\mathrm{G}_{k}+\rho_{\mathrm{m}} \varepsilon+\frac{\partial}{\partial x_{j}}\left[\alpha_{k}\left(\mu_{\mathrm{m}}+\mu_{\mathrm{t}}\right) \frac{\partial k}{\partial x_{j}}\right] \\
\frac{\partial\left(\rho_{\mathrm{m}} u_{i} \varepsilon\right)}{\partial x_{i}}=C_{1 \varepsilon} \frac{\varepsilon}{k} \mathrm{G}_{k}-C_{2 \varepsilon} \rho_{\mathrm{m}} \frac{\varepsilon^{2}}{k}+\frac{\partial}{\partial x_{j}}\left[\alpha_{\varepsilon}\left(\mu_{\mathrm{m}}+\mu_{\mathrm{t}}\right) \frac{\partial \varepsilon}{\partial x_{j}}\right] \\
\mu_{t}=\rho_{\mathrm{m}} c_{\mu} \frac{k^{2}}{\varepsilon}
\end{gathered}
$$

where $\varepsilon$ and $G_{k}$ denote the turbulent kinetic energy dissipation rate and turbulent kinetic energy generation term, respectively. The empirical constants are $\alpha_{k}=1.39, \alpha_{\varepsilon}=1.39$, and $c_{\mu}=0.09$.

The compressibility should be considered for the cavitating flow consisting of liquid and vapor. To take into account the effects of the compressibility, a filter function can be introduced to the turbulence model [11].

$$
\mu_{\mathrm{t}}=\rho_{\mathrm{m}} c_{\mu} \frac{k^{2}}{\varepsilon} F,
$$

where $F$ is the filter function of the filter size $(\Delta)$, the turbulent kinetic energy and dissipation rate, as shown below

$$
F=\operatorname{Min}\left[1, \frac{\Delta \varepsilon}{k^{3 / 2}}\right] \text {. }
$$

In order to ensure the filtering process, the filter size in the present study is chosen to be larger than the largest grid scale employed in the computation. According to eq. (10), the turbulent structure smaller than the filter size will not be resolved.

In the calculation, a commercial CFD code Fluent coupled with the RNG $k-\varepsilon$ turbulence model modified by a user defined file was used. The SIMPLEC algorithm was used to solve the above steady flow governing equations. The boundary conditions are as follows: the constant flow velocity at the inlet and the pressure at the outlet, the standard wall functions were imposed over the impeller blades and sidewalls, the volute casing and the inlet and outlet pipe walls in order to work with the filter function. The pressure at pipe outlet was decreased step by step with a convergence at every given pressure value. The multiple rotating reference frame (MRF) was applied to couple the rotation and station domains.

\section{Results and analysis}

The design point of the centrifugal pump is as follows: the volume flow rate $Q=25 \mathrm{~m}^{3} / \mathrm{h}$, the rotational speed $n=1450$ $\mathrm{r} / \mathrm{min}$, the head $H=7 \mathrm{~m}$. Figure 1 shows the computation domain of the centrifugal pump.

The pump head is the difference in the total energies at 
(a)

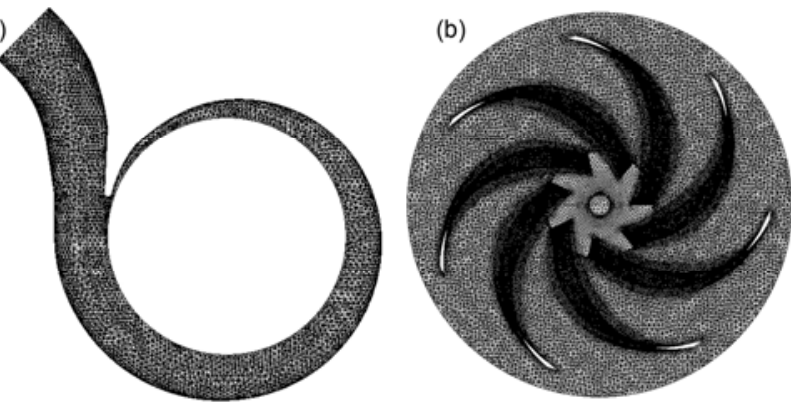

Figure 1 Computation domain of the centrifugal pump. (a) Volute; (b) impeller.

pump inlet and outlet, and it is defined as follows:

$$
\begin{aligned}
H= & {\left[\sum_{i=1}^{n_{o}}\left(\frac{p}{\rho_{l} g}+\frac{u^{2}}{2 g}\right)_{i} u_{i} \cdot A_{i} / \sum_{i=1}^{n_{o}} u_{i} \cdot A_{i}\right]_{\text {out }} } \\
& -\left[\sum_{i=1}^{n_{i}}\left(\frac{p}{\rho_{l} g}+\frac{u^{2}}{2 g}\right)_{i} u_{i} \cdot A_{i} / \sum_{i=1}^{n_{i}} u_{i} \cdot A_{i}\right]_{\text {in }},
\end{aligned}
$$

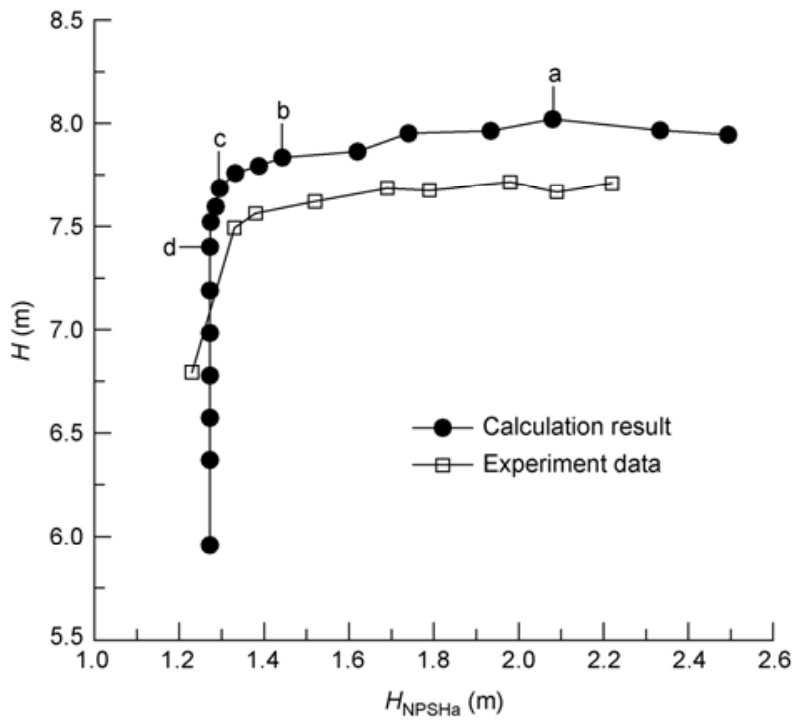

Figure 2 The experimental and numerical results of the $H$ varying with $H_{\mathrm{NPSHa}}$
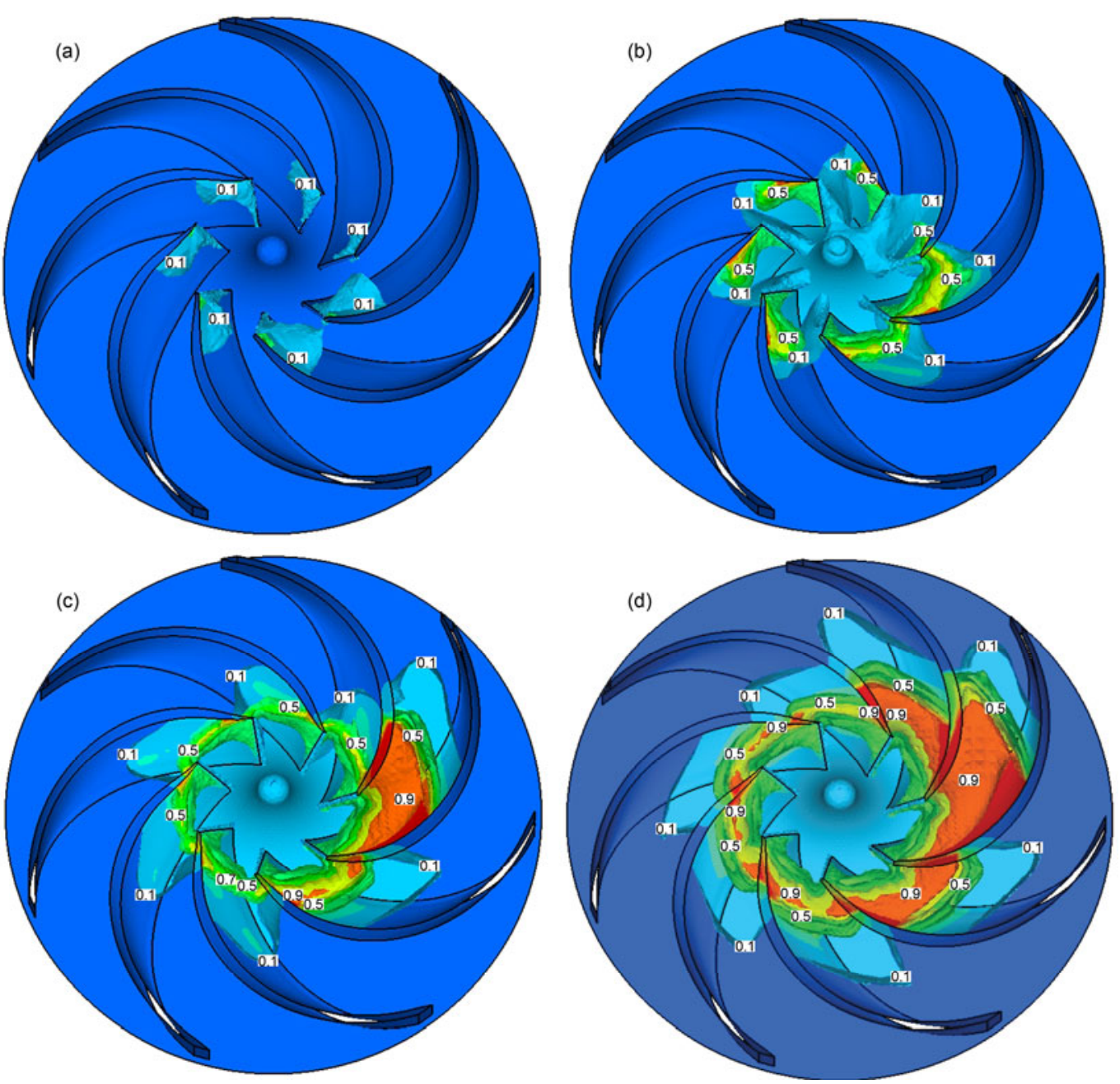

Figure 3 The cavity volume fraction in centrifugal pump impeller. (a) $H_{\mathrm{NPSHa}}=2.08 \mathrm{~m}$; (b) $H_{\mathrm{NPSHa}}=1.44 \mathrm{~m}$; (c) $H_{\mathrm{NPSHa}}=1.30 \mathrm{~m}$; (d) $H_{\mathrm{NPSHa}}=1.27 \mathrm{~m}$. 
where $n_{\mathrm{i}}$ and $n_{\mathrm{o}}$ are the node numbers at the computation domain inlet and outlet, and $A_{i}$ denotes the unit area vector.

The $H_{\mathrm{NPSH}}$ is the difference between the total energy and vaporization energy for unit weight of the fluid at the pump inlet. The higher the magnitude of the $H_{\mathrm{NPSHa}}$ is, the less likely the cavating flow is to take place in the pump. The value of $H_{\mathrm{NPSHa}}$ at which the head drops by $3 \%$ is defined as the net positive suction head critical $\left(H_{\mathrm{NPSHc}}\right)$. The $H_{\mathrm{NPSHa}}$ is defined as follows:

$$
H_{\mathrm{NPSHa}}=\frac{p}{\rho_{1} g}+\frac{u^{2}}{2 g}-\frac{p_{\mathrm{v}}}{\rho_{l} g} \text {. }
$$

Figure 2 shows the experimental and numerical results of the head varying with $H_{\mathrm{NPSH}}$ at $Q=19.80 \mathrm{~m}^{3} / \mathrm{h}, 79 \%$ of designed flow rate. The cavitation performance of centrifugal pump was measured using the test apparatus set up by Tan et al. [12] consisting of an outlet section, a pump section and a water supply section with the same test method. The numerical results are in good agreement with the experimental data, especially the sudden decline in $H$ corresponding to the $H_{\mathrm{NPSH}}$ decrease. The deviation of $H_{\mathrm{NPSHc}}$ between the experiment and calculation is $2.25 \%$.

Figure 3 shows the variation of cavity volume fraction with different $H_{\mathrm{NPSH}}$, i.e $2.08,1.44,1.30$, and $1.27 \mathrm{~m}$, corresponding to the letters a , b, c and d in Figure 2, in centrifugal pump impeller at $Q=19.80 \mathrm{~m}^{3} / \mathrm{h}$. The cavity inception and development in the pump are clearly obtained. The cavity first appears on the suction side near the blade leading edge, at which the pressure in the region near the shroud is smaller than that of the hub due to the centrifugal force, so the cavitation area becomes larger from hub to shroud, as shown in Figure 3(a). With the decrease in $H_{\mathrm{NPSH}}$, the cavity expends to the impeller channels and blocks the flow. Hence, the pump work ability will be confined, and the pump head begins to drop, as shown in Figure 3(b). In Figure $3(\mathrm{c})$, the cavitation bubbles become more and more larger resulting in a sudden fall in the pump head. At this condition, the centrifugal pump cannot work normally, and the large pressure fluctuation and acoustic emission due to the bubble collapse may damage the impeller blades. As the $H_{\mathrm{NPSH}}$ decreases further, bubbles occupy the whole impeller channels and the pump head reduces by $30 \%$, as shown in Figure 3(d). The distributions of the cavitation on blade or in channel are asymmetrical because of the pump volute.

\section{Conclusions}

An improved computation model with modified RNG $k-\varepsilon$ turbulence model and full cavitation model is used to calculate the cavitating flow in a centrifugal pump at a low flow rate. The effects of the compressibility of mixture fluid were included by introducing a filter function for the RNG $k-\varepsilon$ turbulence model. The calculated results of pump head varying with $H_{\mathrm{NPSHa}}$ are consistent with the measurements, and the cavitation variations with $H_{\mathrm{NPSHa}}$ are discussed. Both the cavitation characteristics inside the impeller and the hydraulic performances show that the proposed method is applicable to simulate the cavitating flow in centrifugal pumps at off-designed conditions.

This work was supported by the National Natural Science Foundation of China (51176088 and 51179090), the National Basic Research Program of China (2009CB724304) and the Open Research Fund Program of State Key Laboratory of Hydroscience and Engineering (sklhse-2012-E-02).

1 Wang H, Zhu B S. Numerical prediction of impact force in cavitating flows. J Fluids Eng, 2010, 132: 1013011-1013019

2 Brennen C E. Hydrodynamics of Pumps. New York: Oxford University Press, 1994

3 Medvitz R B, Kunz R F, Boger D A, et al. Performance analysis of cavitating flow in centrifugal pumps using multiphase CFD. J Fluids Eng, 2002, 124: 377-383

4 Coutier-Delgosha O, Fortes-Patella R, Reboud J, et al. Experimental and numerical studies in a centrifugal pump with two-dimensional curved blades in cavitating condition. J Fluids Eng, 2003, 125: 970-978

5 Pouffary B, Patella R F, Reboud J L, et al. Numerical simulation of 3D cavitating flows: Analysis of cavitation head drop in turbomachinery. J Fluids Eng, 2008, 130: 0613011-06130110

6 Ding H, Visser F, Jiang Y, et al. Demonstration and Validation of a 3D CFD Simulation Tool Predicting Pump Performance and Cavitation for Industrial Applications. J Fluids Eng, 2011, 133: 011101101110114

7 Kunz R F, Boger D A, Stinebring D R, et al. A preconditioned NavierStokes method for two-phase flows with application to cavitation prediction. Comput Fluids, 2000, 29: 849-875

8 Singhal A K, Athavale M M, Li H, et al. Mathematical basis and validation of the full cavitation model. J Fluids Eng, 2002, 124: 617-624

9 Wang G Y, Senocak I, Shyy W. Dynamics of attached turbulent cavitating flows. Prog Aerospace Sci, 2001, 37: 551-581

10 Coutier-Delgosha O, Stutz B, Vabre A, et al. Analysis of cavitating flow structure by experimental and numerical investigations. J Fluid Mech, 2007, 578: 171-222

11 Johansen S T, Wu J, Shyy W. Filter-based unsteady RANS computations. Int J Heat Fluid Fl, 2004, 25: 10-21

12 Tan L, Cao S L, Gui S B. Hydraulic design and pre-whirl regulation law of inlet guide vane for centrifugal pump. Sci China Tech Sci, 2010, 53: 2142-2151

Open Access This article is distributed under the terms of the Creative Commons Attribution License which permits any use, distribution, and reproduction in any medium, provided the original author(s) and source are credited. 